\title{
Deformation of Elastic Damping Bodies, Hysteresis Friction
}

\author{
Leon Makharadze, Levan Gavasheli* \\ Academy of Physical and Mathematical Sciences, Georgia.
}

How to cite this paper: Leon Makharadze, Levan Gavasheli. (2020) Deformation of Elastic Damping Bodies, Hysteresis Friction. Journal of Applied Mathematics and Computation, 4(3), 57-63.

DOI: $10.26855 /$ jamc.2020.09.002

Received: June 8, 2020

Accepted: June 30, 2020

Published: July 16, 2020

*Corresponding author: Levan Gavasheli, Academy of Physical and Mathematical Sciences, Georgia.

Email: levan_gavasheli@yahoo.com

\begin{abstract}
The article considers the issue of deformation of elastically damping bodies, taking into account the influence of hysteresis energy losses of forced vibrations. It was found than the longitudinal vibrations of nonlinear mechanical systems with distributed parameters mainly depend on the generalized stiffness coefficient, the natural frequency of the system, and on the increment of the driving force. A monotonic change in the stiffness coefficient of elastic-damping materials during hysteretic friction leads to an automatic change in the natural frequency as a whole.
\end{abstract}

\section{Keywords}

Deformation, hysteresis friction, forced vibrations, longitudinal vibrations, stiffness coefficient, generalized stiffness coefficient, natural frequency

\section{Introduction}

As you know, in the cyclical deformation of elastic-damping bodies, there is a violation of the law of Hook, expressed in the appearance of the loop of hysteresis, as shown in the Figure 1, 2, and 3. It shows the static characteristics of elastic-damping materials monotonously increasing (Figure 1, 2) and monotonously decreasing (Figure 1, 3) rigidity [1]. From rice. 1, 2 and 3, it can be seen as static and respectively dynamic characteristics of elastic-damping materials (metal rubber, technical rubber, cork mass, metal bellows, etc.) [2] [3]. They have a non-linear (parabolic) characteristic, the mathematical description of which is very difficult. In order to quantify the rate of fading of free and forced fluctuations of mechanical systems in the presence of the above elastic-damping materials, for the first time a mathematical description of the shape of the loops of hysteresis, which are shown in the rice, is proposed for the first time. 1, 2 and 3 and are described in equations (1), (2), (3):

$$
\begin{aligned}
& f\left(x, \frac{d x}{d t}\right)=\frac{1}{2}\left[\frac{x^{2}}{2 P}\left(\operatorname{sgn} \frac{d x}{d t}+1\right)-\sqrt{2 P x}\left(\operatorname{sgn} \frac{d x}{d t}-1\right)\right] \\
& f_{1}\left(x, \frac{d x}{d t}\right)=\frac{1}{2}\left[P_{1} x^{2}\left(\operatorname{sgn} \frac{d x}{d t}+1\right)-P_{2} x^{3}\left(\operatorname{sgn} \frac{d x}{d t}-1\right)\right] \\
& f_{2}\left(x, \frac{d x}{d t}\right)=\frac{1}{2}\left[\sqrt{P_{1} x}\left(\operatorname{sgn} \frac{d x}{d t}+1\right)-\sqrt[4]{P_{2} x}\left(\operatorname{sgn} \frac{d x}{d t}-1\right)\right] .
\end{aligned}
$$

Here, the coefficients $P, P_{1}, P_{2}$ which are determined experimentally, depend on the stiffness and elastic modulus of the starting material. From the expressions $(1,2,3)$, it is seen that in the presence of energy dissipation, the frequency characteristics of the system change insignificantly. 
Obviously, under load $\frac{d x}{d t}>0$, respectively $\operatorname{sgn} \frac{d x}{d t}=1$, during unloading $\frac{d x}{d t}<0$ and $\operatorname{sgn} \frac{d x}{d t}=-1$.

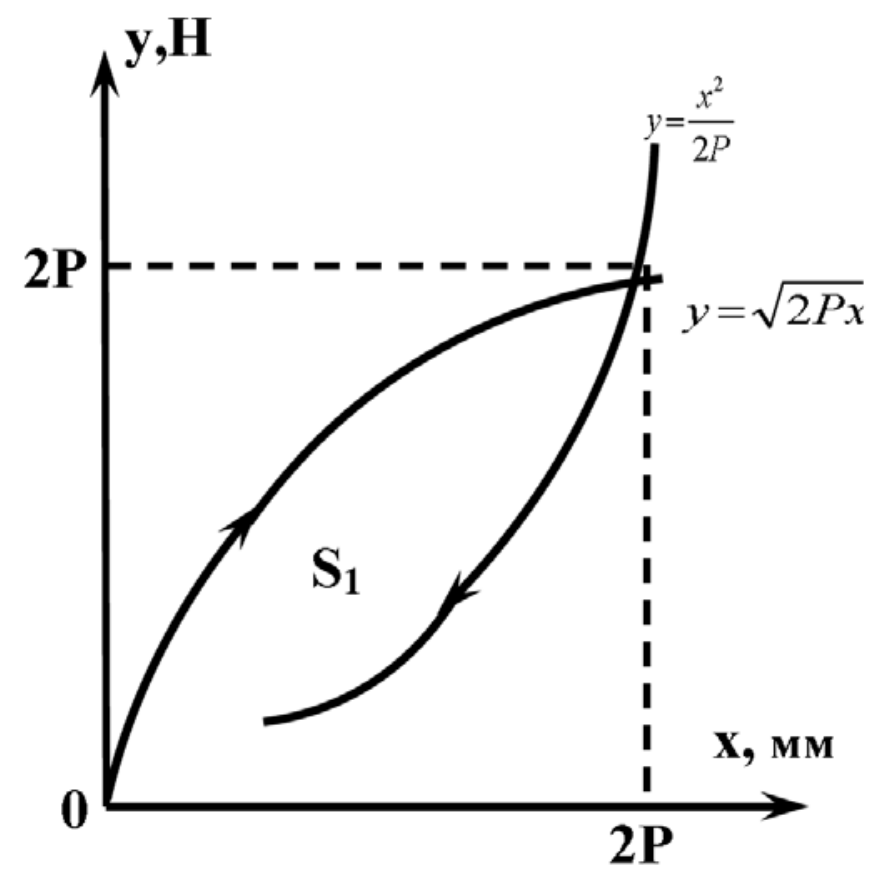

Figure 1. Hysteresis friction of parabolic type.

$$
S_{1}=\int_{0}^{2 P}\left(\sqrt{2 P x}-\frac{x^{2}}{2 P}\right) d x=\frac{4}{3} P^{2}
$$

The hysteresis area determines the energy that is dissipated in one cycle of oscillations per unit volume of the material.

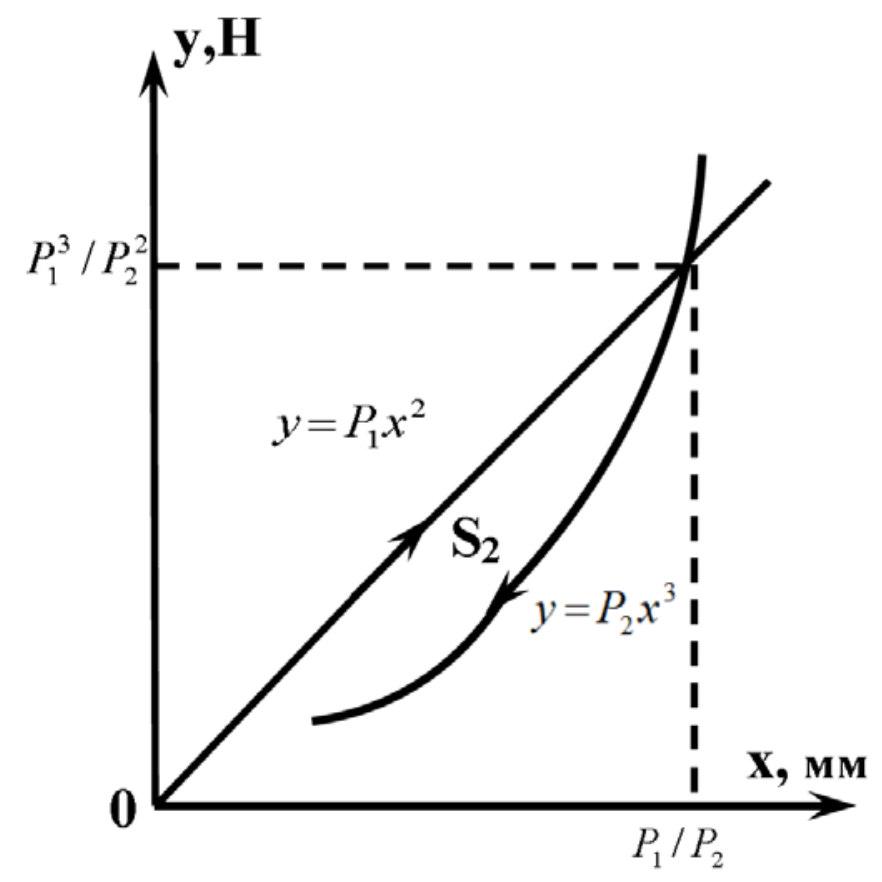

Figure 2. Hysteresis friction of monotonically increasing stiffness. 


$$
S_{2}=\int_{0}^{P_{1} / P_{2}}\left(P_{1} x^{2}-P_{2} x^{3}\right) d x=\frac{P_{1}}{12} \cdot\left(\frac{P_{1}}{P_{2}}\right)^{3} .
$$

Similarly, the $S_{2}$ hysteresis area corresponds to the energy dissipated in one oscillation cycle, with a monotonously increasing stiffness.

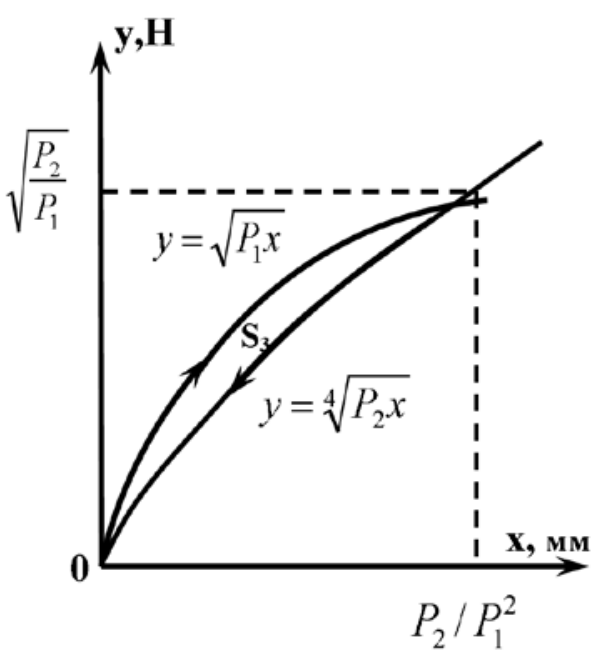

Figure 3. Hysteresis friction of monotonically decreasing stiffness.

$$
S_{3}=\int_{0}^{P_{2} / P_{1}^{2}}\left(\sqrt{P_{1} x}-\sqrt[4]{P_{2} x}\right) d x=\frac{2}{3} \sqrt[6]{\frac{P_{2}^{4}}{P_{1}^{5}}}-\frac{4}{5} \sqrt{\frac{P_{2}^{3}}{P_{1}^{5}}} \approx 1-\frac{4}{5} \sqrt[3]{\frac{P_{2}^{3}}{P_{1}^{5}}},
$$

where the $S_{3}$ hysteresis area determines the dissipated energy, with monotonously decreasing stiffness. Figures 1, 2, 3 show hysteresis loops of a parabolic type in the coordinate axes of stress $\mathrm{y}, \mathrm{H}$ and is the strain $\mathrm{x}, \mathrm{mm}$. As they can be seen, the $S_{1}, S_{2}, S_{3}$ are independent of the rate of deformation, i.e. on the frequency of the process, but depend on the amplitude of the deformation. Here $P, P_{1}, P_{2}$, the constants are determined experimentally at special facilities, in particular at a universal stand with a pulsator.

\section{Metods}

To determine the law that describes the damping of oscillations during hysteresis friction, we use the energy balance equation. Since the kinetic energy is zero at the beginning and end of the cycle in question, the change in the total energy of the system is determined by the change in potential energy. When calculating this change, it is necessary to take into account the difference between the largest deviations (Figure 4) $P(0)$ and $P(\mathrm{~T}) \quad$ [4].

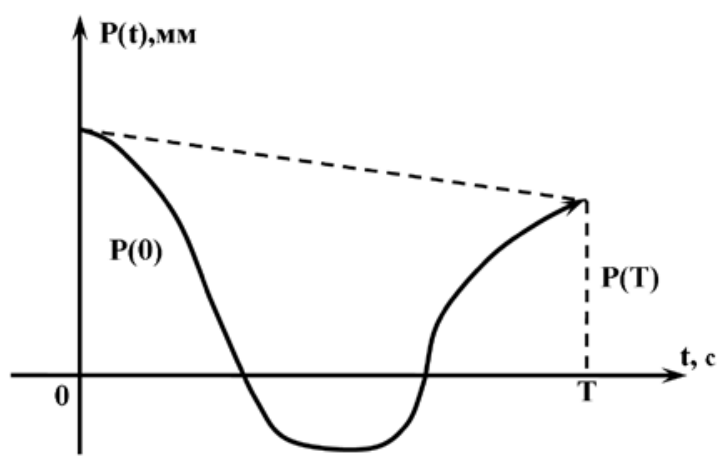

Figure 4. The greatest deviations of the potential energy for one period $(0, T)$ of oscillations.

Accordingly, at the beginning of the cycle, the potential energy is 


$$
\Pi(0)=\frac{1}{2} c P^{2}(0),
$$

where $\mathrm{c}$ is the generalized stiffness coefficient, $\mathrm{H} / \mathrm{m}$, and at the end of the cycle the energy $P(T)$ is

$$
\Pi(\mathrm{T})=\frac{1}{2} c P^{2}(\mathrm{~T}) \text {, }
$$

The increment of potential energy $\Delta \Pi$ is equal to

$$
\Delta \Pi=\frac{1}{2} c\left[P^{2}(\mathrm{~T})-P^{2}(0)\right] .
$$

The dissipated energy in the first case (Figure 1) does not depend on the oscillation frequency and is taken in the form $E=\frac{4}{3} P^{2}(t)$, equating this energy with a minus sign, the incremental energy in one period, we have

$$
-\frac{4}{3} P^{2}(t)=\frac{1}{2} c\left[P^{2}(\mathrm{~T})-P^{2}(0)\right]
$$

Accordingly, in the second case, we have

$$
-\left[\frac{P_{1}}{12} \cdot\left(\frac{P_{1}}{P_{2}}\right)^{3}\right]=\frac{1}{2} c\left[P^{2}(\mathrm{~T})-P^{2}(0)\right],
$$

and therefore, in the third case, we have

$$
-\left[\frac{2}{3} 6 \sqrt{\frac{P_{2}^{3}}{P_{1}^{5}}}-\frac{4}{5}{\sqrt{\frac{P_{2}^{3}}{P_{1}^{5}}}}^{3}\right]=\frac{1}{2} c\left[P^{2}(\mathrm{~T})-P^{2}(0)\right] .
$$

In expression (9) $\left[P^{2}(\mathrm{~T})-P^{2}(0)\right]=[P(\mathrm{~T})+P(0)] \cdot[P(\mathrm{~T})-P(0)]$, we approximately $[P(\mathrm{~T})+P(0)]$ replace $2 P(0)$, and $[P(\mathrm{~T})-P(0)]$ - through $\Delta P$, then we have $-\frac{4}{3} P^{2}(t)=c P(0) \Delta P$ or the equation in finite differences takes the form

$$
\Delta P=-\frac{4 P(t)}{3 c},
$$

the differential equation for the upper envelope has the form

$$
\Delta P=T \cdot \frac{d P}{d t}=\frac{2 \pi}{k} \cdot \frac{d P}{d t} .
$$

After integrating equation (14) under the initial condition $P(0)=P_{0}$, we obtain

$$
\frac{d P}{d t}=-\frac{4 P(t)}{3 c}: \frac{2 \pi}{k}=-\frac{2 k P(t) I(1)}{3 \pi c},
$$

where I is the Euler integral of the second kind; $\mathrm{k}$ is the natural frequency of the system.

$$
I=\frac{2^{n-2} n^{2} \Gamma\left(\frac{n}{2}\right)}{n(n+1) \Gamma(n)},
$$

where $\Gamma$ is the gamma function in our case $\mathrm{n}=1$, respectively $I=\frac{\pi}{4}$, i.e. we have

$$
\frac{d P}{d t}=-\frac{2 k \cdot \frac{\pi}{4}}{3 \pi c} P(t)=-\frac{k}{6 c} \cdot P(t)=-h P(t),
$$


where $h=\frac{k}{6 c}$. The solution of equation (16) has the form

$$
P(t)=P_{0} \cdot e^{-h t}
$$

Thus, the hysteresis friction of the upper envelope (as a continuous curve) has the form of an exponential characteristic, respectively, the motion graphs will be uneven and depend only on the generalized stiffness coefficient c and the natural frequency of the system $\mathrm{k}$, in this case the logarithmic decrement does not depend on the amplitude of oscillations and remains unchanged in the process of oscillations of the system as a whole, in addition, with increasing rigidity, the amplitude of oscillations of the systems decreases.

In the second case, the energy balance equation has the form

$$
\begin{aligned}
& -\left[\frac{P_{1}}{12} \cdot\left(\frac{P_{1}}{P_{2}}\right)^{3}\right]=c \cdot P(0) \Delta P, \\
& \Delta P=-\frac{\left[\frac{P_{1}}{12} \cdot\left(\frac{P_{1}}{P_{2}}\right)^{3}\right]}{c P(0)} .
\end{aligned}
$$

If we approximately assume that $P_{1}=1,4 P_{2}$, then we have $\left(\frac{P_{1}}{P_{2}}\right)^{3} \approx 2,4$, i.e

i.e.

$$
\Delta P=-\frac{P(t)}{5 c P_{0}}=\frac{2 \pi}{k} \cdot \frac{d P(t)}{d t}, \frac{d P(t)}{d t}=-\frac{P(t)}{5 c P_{0}}: \frac{2 \pi}{k}=-\frac{P(t) k I(1)}{10 c P_{0} \pi} . I(1)=\frac{\pi}{4},
$$

$$
\frac{d P(t)}{d t}=-\frac{k}{40 c P_{0}} P(t)=-h_{1} P(t)
$$

Where $h_{1}=\frac{k}{40 c P_{0}}$. Similarly, in the first case, the solution of equation (19) has the form

$$
P(t)=P_{0} \cdot e^{-h_{1} t}
$$

In this case, the coefficient $\boldsymbol{h}_{1}$ depends on the rigidity of the system $c$, on the natural frequency $K$ and on the initial values of $P_{0}$. The shape of the upper envelope will also be exponential and the motion graphs will be unequal and, with an increase in the initial value of the oscillation amplitude $P_{0}$, will accordingly increase.

In the third case, the energy balance equation has the form

$$
-\left[\frac{2}{3} 6 \sqrt{\frac{P_{2}^{4}}{P_{1}^{5}}}-\frac{4}{5} \sqrt{\frac{P_{2}^{3}}{P_{1}^{5}}}\right]=c P(0) \Delta P .
$$

Approximately we have

$$
\begin{aligned}
& -\left[1-1,5 \cdot \frac{1}{P_{1}(t)}\right]=c P_{0} \cdot \Delta P \\
& \text { or }-\left[1-1,5 \cdot \frac{1}{P_{1}(t)}\right]=c P_{0} \cdot \Delta P \\
& P_{1}(t)+c P_{0} \cdot P_{1}(t)=1,5 \text { or } P_{1}(t)\left(1+c P_{0} \Delta P\right)=1,5
\end{aligned}
$$




$$
\begin{aligned}
& \text { or } \Delta P=-\frac{1,5-P_{1}(t)}{c P_{0} \cdot P_{1}(t)}=-\frac{P_{1}(t)-1,5}{c P_{0} \cdot P_{1}(t)}, \quad \frac{d P(t)}{d t}=-h_{2} P(t), \\
& \text { where } h_{2}=\frac{1,5 k}{2 \pi \cdot c \cdot P_{0}} .
\end{aligned}
$$

The solution of equation (11) takes the form

$$
P(t)=-P_{0} \cdot e^{-h_{2} t}
$$

The coefficient $h_{2}=\frac{1,5 k}{2 \pi \cdot c \cdot P_{0}}$ is directly proportional to the natural frequency $k$ and inversely proportional to the stiffness coefficient $c$ and the initial value of the oscillation amplitude of the system $P_{0}$. The motion graphs will be exponential and uneven, in addition, with a decrease in the system stiffness coefficient, the oscillation amplitudes increase.

As the law that determines the damping of oscillations during hysteretic friction confirms, it mainly depends on the stiffness coefficient and on the own frequency of the system, therefore, in order to fully evaluate the effect of hysteretic friction on the damping of longitudinal vibrations of systems with distributed parameters, it is necessary to consider forced vibrations of a long rod located in elastic -damping medium, the intensity of which can be taken in the form $-q x$, where $q$ is the stiffness coefficient of the medium. Differential equations of longitudinal vibrations in such a medium have the form

$$
\frac{E}{\rho} \cdot \frac{\partial^{2} y}{\partial x^{2}}-\frac{q}{\rho S} y-\frac{\partial^{2} y}{\partial t^{2}}=F(x, t),
$$

where $E$ - is the modulus of elasticity of the rod; $\rho$ - - the density of the material of the rod; $S$ - the cross-sectional area of the rod; $F(x, t)$ - generalized driving force.

We are looking for private solutions in the form $y=u(x) \mathrm{v}(t)$. We substitute this expression into (28) and, separating the variables, we arrive at differential equations

$$
\begin{aligned}
& \frac{d^{2} v}{d t^{2}}+P^{2} v(t)=0 ; \\
& \frac{d^{2} u}{d x^{2}}+a^{2} u(x)=F(x, t) ;
\end{aligned}
$$

where $a^{2}=\left(P^{2}-\frac{q}{\rho S}\right) \cdot \frac{\rho}{E} ; P=\sqrt{1+\frac{(a 1)^{2}}{b}} \cdot \sqrt{\frac{q}{\rho S}} ; b=\frac{q 1^{2}}{E S} ; \ell-$ - the length of the rod.

The solution of equation (29) in the form $v(t)=P_{0} \sin (\omega t+\varphi)$ shows that the movement is harmonic oscillations with an angular frequency $\omega$, where the initial conditions $\omega(0)=v_{0} \frac{d v(0)}{d t}=\frac{d v_{0}}{d t}$ respectively

$$
P_{0}=\sqrt{\left(\frac{v_{0}}{P}\right)^{2}+v_{0}^{2}} ; \quad \varphi=\operatorname{arctg} \frac{P v_{0}}{\frac{d v_{0}}{d t}} .
$$

The dynamic effect of the forced force $F(x, t)$, on the rod can be found by integration in the following form:

$$
u(x)=\frac{F(x, t)}{a}-\frac{1}{a} \int_{0}^{t} \frac{\partial F(x, t)}{\partial x} \cos \omega(t-x) d x .
$$

When the force $F(x, t)$ acts on the rods for a sufficiently long period of time (but not impulse), then we have one maximum of the forced force and the relation 


$$
\left|-\frac{1}{a} \int_{0}^{t} \frac{\partial F(x, t)}{\partial x} \cos \omega(t-x) d x\right|<\frac{\frac{\partial F_{\max }(x, t)}{\partial x} \cdot T}{2 a},
$$

where $\frac{\partial F_{\max }(x, t)}{\partial x}$ is the maximum value of the rate of change of force; $T$ is the period of oscillation. The expression $\frac{\partial F_{\max }(x, t)}{\partial x} \cdot T$

$\frac{\partial x}{2 a}$ determines the maximum possible increment of the driving force $F(x, t)$ for a half-period of free oscillations of the system.

If, then $t_{1}<\frac{T}{2}$, the greatest deviation of the system is achieved after the disappearance of the force, then $t_{0}>t_{1}$, we have

$$
u(x)=\frac{F_{0}}{\omega a} \int_{0}^{t_{1}} \sin \omega(t-x) d x=\frac{2 F_{0}}{a} \sin \frac{\omega t_{1}}{2} \sin \omega\left(t-\frac{t_{1}}{2}\right)
$$

the largest deviation is

$$
u_{\max }(x)=\frac{F_{0}}{a} \sin \pi c, c=\frac{t_{1}}{T},
$$

where $F_{0}$ - is the initial value of the driving force.

The characteristic equation for differential equation (30) has the form

$$
\operatorname{tga} 1=-M\left(a 1-\frac{K}{a 1}\right)
$$

where $m=\frac{M}{\rho S 1}$ is the relative mass of the rod, $M$ is the mass of the system as a whole; $K=\frac{q 1^{2}}{E S}-$ dimensionless coefficient of rigidity of the rod.

Provided $\frac{\partial u(l, t)}{\partial t}=0$, we find the first own frequency of the system

$$
\omega_{1}=\sqrt{\frac{q}{\rho S}}<\sqrt{1+\frac{(a+1)^{2}}{b}} \cdot \sqrt{\frac{q}{\rho S}} .
$$

\section{Conclusion}

If the relative mass of the rod decreases, then the own frequency will increase, the first own frequencies correspond to a somewhat special own function - the movement of the entire rod as a rigid whole along its axis, and with the increase of the stiffness coefficient to a certain value, the attenuation rate of the rod oscillations increases, respectively, of the rod in the longitudinal direction decreases. Thus, we can conclude that a monotonic change in the stiffness coefficient of elastically damping materials during hysteretic friction leads to an automatic change in the natural frequency of the system as a whole and, with a wide range of changes in the frequency of the disturbing force, ensure a successful fight against harmful vibrations.

\section{References}

[1] Gavasheli L. Sh. (2006). Theory of vibration protection of nonlinear mechanical systems. Metznireba, Tbilisi, p. 272.

[2] Makharadze L. I. (1996). Protection of hydraulic systems against water hammer. Metznireba, Tbilisi, p. 150.

[3] GavasheliL. Sh., Makharadze L. I. (2016). Transverse damping of main pipelines of hydrotransport systems under the influence of parametric forces. Georgian engineering news, №2, pp. 59-62.

[4] Panovko Ya. G. (1980). Introduction to the theory of mechanical vibrations. Science, Moscow, pp. 140-150. 